subsequently comparing QMVC assessment using these reference equations in two separate cohorts of patients with COPD.

Methods Prediction equations were derived through multiple linear regression in a healthy control (HC) group. Age, gender, height and weight were inputted into the first model (FFMmodel) and fat-free mass (FFM) added for the other (FFM+ model). The prediction equations were then applied to a Primary Care COPD (PCC) group and Complex Care COPD (CCC) group of patients where percentage predicted values were calculated and weakness determined using a threshold of the lower limit of normal.

Results 175 HC subjects (median (IQR) age: 54 (14) years, 31\% male) were recruited. The PCC group comprised 87 patients (median (IQR) age: 68 (9) years, 71\% male, FEV1 62 (20)\% predicted) and the CCC group 189 patients (median (IQR) 66 (12) years, 57\% male, FEV1: 29 (16)\% predicted).

Prediction values for the $\mathrm{HC}$ and PCC were similar between the FFM- and FFM+ models as shown in the table. In the CCC percentage predicted values were lower and there were $11.9 \%$ more classed as weak by the FFM- model compared to the FFM + model.

\begin{tabular}{|c|c|c|c|}
\hline & $\begin{array}{l}\text { Healthy } \\
\text { control }\end{array}$ & $\begin{array}{l}\text { Primary care } \\
\text { COPD }\end{array}$ & $\begin{array}{l}\text { Complex care } \\
\text { COPD }\end{array}$ \\
\hline & $\mathrm{n}=175$ & $\mathrm{n}=87$ & $\mathrm{n}=189$ \\
\hline \multicolumn{4}{|l|}{$\mathrm{FFM}^{-}$Model } \\
\hline \%pred QMVC: & $100.3(24.1)$ & $86.0(22.0)$ & $54.0(16.4)$ \\
\hline Number classed as weak & $6(3.4 \%)$ & $14(16.3 \%)$ & $101(53.2 \%)$ \\
\hline \multicolumn{4}{|l|}{ (\%): } \\
\hline \multicolumn{4}{|l|}{$\mathrm{FFM}^{+}$Model } \\
\hline \% pred QMVC: & $100.2(24.1)$ & 86.7 (20.6) & $59.2(17.8)$ \\
\hline Number classed as weak & $8(4.6 \%)$ & $10(11.6 \%)$ & $78(41.3 \%)$ \\
\hline
\end{tabular}

Conclusion The inclusion of fat-free mass did not significantly alter prediction of muscle weakness in the healthy cohort. In the COPD cohorts, including FFM in the model altered the proportion classified as having muscle weakness, most notably in the CCC cohort. This is likely to be due to a higher prevalence of muscle wasting in this population which resulted in an underestimate of predicted strength when muscle mass is included in the model.
P48

\section{RESEARCH BAL USING SINGLE USE DISPOSABLE} BRONCHOSCOPE

${ }^{1 S}$ Zaidi, ${ }^{1} \mathrm{~A}$ Collins, ${ }^{2} \mathrm{~K}$ Davies, 'A Wright, ${ }^{2} \mathrm{~A}$ Ganguli, ${ }^{1} \mathrm{E}$ Mitsi, ${ }^{1} \mathrm{f}$ Reine, ${ }^{1} \mathrm{~J}$ Owugha, ${ }^{3} S$ Gordon, 'D Ferreira, 'J Rylance. 'Liverpool School of Tropical Medicine, Liverpool, UK; ${ }^{2}$ Royal Liverpool University Hospital, Liverpool, UK; ${ }^{3}$ Malawi-Liverpool- Wellcome Trust, Clinical Research Programme, Blantyre, Malawi

\subsection{6/thoraxjn--2016-209333.191}

Background Broncho alveolar lavage (BAL) is widely used for investigative research to study innate, cellular and humoral immune responses, and in early phase drug trials. Conventional (multiple use) flexible bronchoscopes have time and monetary costs associated with cleaning, and may also carry a small risk of cross infection. Single use bronchoscopes may provide an alternative, but have not been evaluated in this context.

Methods Healthy volunteers underwent bronchoscopy on a daycase clinical research unit using the Ambu ${ }^{\circledR}$ Scope single-use flexible intubation bronchoscope. The bronchoscopy protocol was identical to previous studies using multiple-use equipment: fasted volunteers had local anaesthesia to the nasopharynx, and were intubated with further sequential local anaesthetic (2\% lidocaine throughout). Lavage was performed from a sub segmental bronchus within the right middle lobe. A total of $200 \mathrm{ml}$ of warmed normal saline divided into four aliquots. Fluid was aspirated using handheld suction. Supplemental oxygen was used to maintain saturations above $90 \%$ throughout the procedure. The lab processing of BAL was identical to earlier studies. BAL volume was recorded, mucus plugs removed by filtration through a double layered gauze swab into sterile centrifuge tubes. The cells were pelleted by centrifugation and washed by vortexing in 50 $\mathrm{mls}$ of cold normal saline, then re-suspended in culture medium for differential counting and viability staining with trypan blue stain.

Results Ten volunteers, (mean age 23 years, 6 male) participated. The procedure was well tolerated by all the participants and all were carried out by two operators. The results were compared to 50 (mean age 23, 14 male) procedures done using the conventional scope by the same two operators. The total volume yield was significantly higher in the disposable group mean (SD) 149 mls (24.6) compared to $123 \mathrm{mls}$ (20.6) p $=0.0007$ Mann-Whitney Test. The total cell yield and viability were similar in both groups, with no significant differences.

Conclusions BAL using single use bronchoscopes are safe with no risk of cross infection, and well tolerated, with potentially reduced side effects post procedure such as pleuritic chest pain and cough as the volume yield is better. The cell yield and viability are comparable to the conventional bronchoscopes. 


\section{BAL Yield}

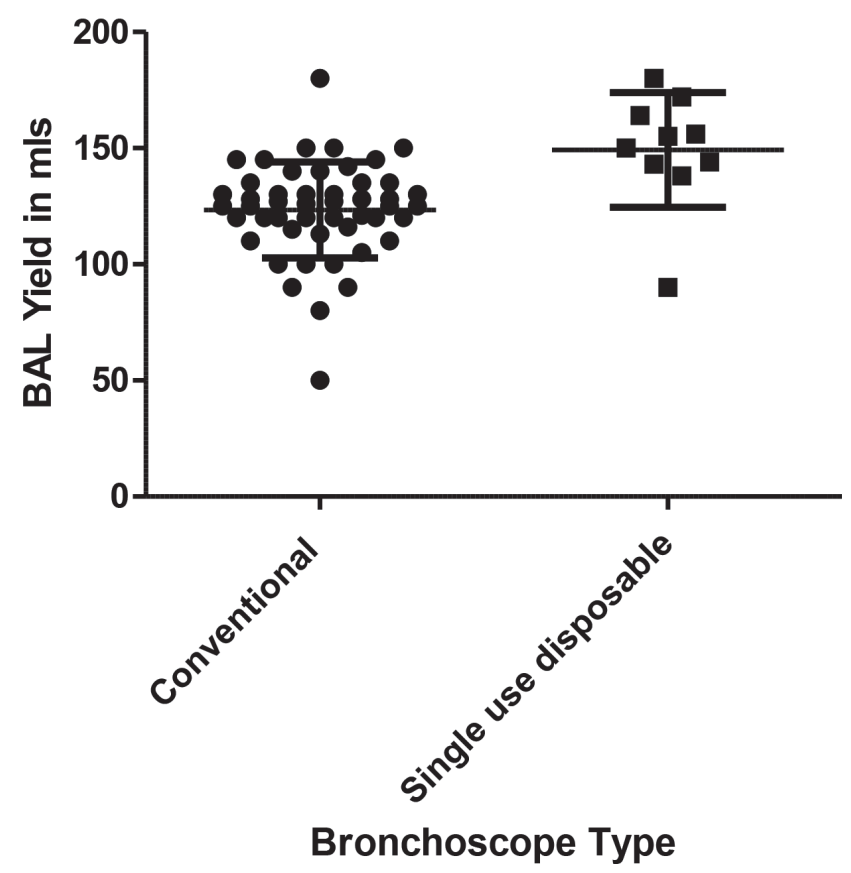

Abstract P48 Figure 1 Graph showing total BAL volume yield from conventional and disposable procedures

\section{P49 DOES VALSALVA MANOEUVRE REDUCE THE RISK OF COMPLICATIONS IN CT-GUIDED LUNG BIOPSIES?}

AE Syed, I Syed, R Ahmed, O Parvu, L Pancione, A Alvi, K Novacic, R Akram. Barking, Havering and Redbridge University Hospitals NHS Trust, Essex, UK

\subsection{6/thoraxjnl-2016-209333.192}

Introduction Multiple complications in CT-guided lung biopsy have been described with pneumothorax known to be the commonest. ${ }^{1}$ This often leads to patients being admitted to hospital for observation or even drainage.

We hypothesised that increased intrathoracic pressure may reduce complications and a comparative retrospective cohort study was performed with the types and rates of complications recorded in patients instructed to perform a Valsalva manoeuvre versus those who were not.

Methods Patients who underwent CT-guided lung or pleural biopsies performed by multiple operators between January 2005 and December 2014 at Queens Hospital, Essex, UK, were retrospectively identified. Information from RIS reports and images from PACS were analysed. Patients were stratified into two groups, those who undertook Valsalva at time of biopsy and those who did not. Complication rates were assessed for haemoptysis, haemothorax and pneumothoraces including those requiring chest drain insertion. Statistical analysis was performed using Chi square test.

Results 791 procedures were performed over 10 years, 420 patients undertook Valsalva manoeuvre with mean ages 70.1 years \pm SD 12.54: range 21-93. The other 371 patients did not undertake a Valsalva manoeuvre. Their mean ages were 71.1 years \pm SD 11.52: range 27-91.

In total, 119 patients had complications post-procedure: 64 in the non-Valsalva group vs 55 in the Valsalva group $(17.25 \%$ vs $13.10 \%, \mathrm{p}=0.05)$.
Rates of haemoptysis were significantly reduced with Valsalva (2.16\% vs $0.24 \%, p=0.006)$. Pneumothorax requiring chest drain $(2.70 \%$ vs $1.43 \%, \mathrm{p}=0.10)$ and those managed conservatively $(12.40 \%$ vs $11.19 \%, \mathrm{p}=0.30)$ were higher in the non-Valsalva group.

Rate of haemothorax $(0.24 \%$ vs $0 \%, \mathrm{p}=1)$ was greater in the Valsalva group.

Conclusion Our study shows that Valsalva manoeuvre at the time of biopsy helps reduce the rate of complications, with a statistically significant decrease in rate of haemoptysis. Rates of pneumothoraces requiring chest drain insertion and other pneumothoraces were also reduced.

The differences could be explained by physiological changes in pulmonary wedge pressure and positive end expiratory pressure brought about by increased intrathoracic pressure following Valsalva manoeuvre.

\section{REFERENCE}

1 Manhire A, Charig M, Clelland C, et al. Guidelines for radiologically guided lung biopsy. British thoracic society guidelines. Thorax 2003;58:920-936.

\section{P50 CHANGING USE OF CT PULMONARY ANGIOGRAPHY IN A UK TERTIARY HOSPITAL OVER A 6-YEAR PERIOD}

${ }^{1} \mathrm{R}$ Ratnakumar, 'JP Corcoran, ${ }^{1} \mathrm{~A}$ Talwar, ${ }^{1} \mathrm{RJ}$ Hallifax, ${ }^{1} \mathrm{I}$ Psallidas, ${ }^{1} \mathrm{JM}$ Wrightson, ${ }^{2} \mathrm{FV}$ Gleeson, ${ }^{1} \mathrm{NM}$ Rahman. 'Oxford Centre for Respiratory Medicine, Oxford University Hospitals NHS Foundation Trust, Oxford, UK; ${ }^{2}$ Department of Radiology, Oxford University Hospitals NHS Foundation Trust, Oxford, UK

\subsection{6/thoraxjnl-2016-209333.193}

Background CT pulmonary angiography (CTPA) is widely regarded as the gold standard test for suspected pulmonary embolism (PE) and is increasingly used as an all-inclusive diagnostic tool for patients with symptoms of acute cardio-respiratory pathology. This has led to an increase in the number of CTPA scans, but no clear reduction in mortality from pulmonary embolic disease. CTPA may be of benefit in making other clinically significant (non-PE) diagnoses, but increased use of CTPA has resource and ionising radiation implications.

Method We reviewed records of patients undergoing CTPA in our centre during the same 6-week reference period in each year from 2009 to 2014 to ascertain the number of scans positive for $\mathrm{PE}$ and/or other diagnoses. The clinical relevance of "other diagnoses" was evaluated, as well as whether "other diagnoses" were evident on earlier investigations such as chest X-ray.

Results Patient records associated with 1882 scans were reviewed. The use of CTPA increased by two-thirds over the 6year study period, with the likelihood of acute PE being diagnosed remaining unchanged (Table 1). Other diagnoses were seen on more than $80 \%$ of scans; the majority were either not therapeutically relevant or potentially evident from previous investigations.

Conclusion Our evaluation of experience in a stable population has demonstrated increased use of CTPA scanning with no change in the diagnostic yield for acute PE. The percentage of patients with additional abnormalities has remained stable, implying the population under investigation is similar. It is uncertain if the increase in the incidence of PE is due to detection of previously undetected emboli, and whether increasing use has reduced mortality from PE. Findings also confirm significant use to screen for other causative pathology. Further work should assess the character of the emboli and the overall impact on morbidity and mortality from increasing CTPA use. 